\title{
The on-board calibration system of the X-ray Imaging Polarimetry Explorer (XIPE)
}

\author{
Silvia Zane ${ }^{\mathrm{a}}$, Berend Winter ${ }^{\mathrm{a}}$, Craig Theobald ${ }^{\mathrm{a}}$, Theodorus Theodorou ${ }^{\mathrm{a}}$, Michele Pinchera ${ }^{\mathrm{b}}$, Fabio \\ Muleri $^{\mathrm{c}}$, Francesca Spada ${ }^{\mathrm{b}}$, Carmelo Sgro ${ }^{\mathrm{b}}$, Davide Zanetti ${ }^{\mathrm{b}}$, Hua Feng ${ }^{\mathrm{d}}$, Ettore del Monte ${ }^{\mathrm{c}}$, Alda \\ Rubinic $^{c}$, Paolo Soffitta ${ }^{\mathrm{c}}$, Ronaldo Bellazzini ${ }^{\mathrm{b}}$,Enrico Bozzo ${ }^{\mathrm{e}}$, Vadim Burwitz ${ }^{\mathrm{f}}$, Alberto J. Castro \\ Tirado $^{\mathrm{g}}$, Enrico Costa ${ }^{\mathrm{c}}$, Szymon Gburek ${ }^{\mathrm{h}}$, Rene Goosmann ${ }^{\mathrm{i}}$, Vladimir Karas ${ }^{1}$, Giorgio Matt ${ }^{\mathrm{m}}$, \\ Kirpal Nandra ${ }^{\mathrm{f}}$, Mark Pearce ${ }^{\mathrm{n}}$, Juri Poutanen ${ }^{\mathrm{o}}$, Victor Reglero ${ }^{\mathrm{p}}$, Maria Dolores Sabau ${ }^{\mathrm{q}}$, Andrea \\ Santangelo $^{\mathrm{r}}$, Giampiero Tagliaferri ${ }^{\mathrm{s}}$, Christopher Tenzer ${ }^{\mathrm{r}}$, Jacco Vink ${ }^{\mathrm{t}}$, Martin Weisskopf ${ }^{\mathrm{u}}$ \\ a Mullard Space Science Laboratory, University College London, Holmbury St Mary, Dorking, \\ Surrey, RH5 6NT, UK; ' 'Istituto Nazionale di Fisica Nucleare, Largo B. Pontecorvo 3, I-56127 \\ Pisa, Italy; ' INAF-IAPS, Via del Fosso del Cavaliere 100, I-00133 Roma, Italy; ${ }^{\mathrm{d} D e p a r t m e n t ~ o f ~}$ \\ Engineering Physics and Center for Astrophysics, Tsinghua University, Beijng, 10084, China; \\ eUniversity of Geneva, ISDC, Switzerland; fMax-Planck-Institut fur extraterrestrische Physik \\ Germany, MPE, Germany; ' IAA, CSIC, Spain; hice Research Centre, PAS, Poland; \\ i Observatoire Astronomique de Strasbourg, France; ${ }^{1}$ Astronomical Institute of the Academy of \\ Sciences Czech Republic; mUniversity Roma 3, Italy; ${ }^{n} \mathrm{KTH}$ Royal Institute of Technology \\ Sweden; 'University of Turku Finland; ' University of Valencia, Spain; ${ }^{9}$ INTA, Spain; ${ }^{r}$ IAAT, \\ University of Tuebingen, Germany; ' $\mathrm{INAF-Osservatorio} \mathrm{Astronomico} \mathrm{di} \mathrm{Brera,} \mathrm{Merate,} \mathrm{Italy;}$ \\ tUniversity of Amsterdam, Anton Pannekoek Institute/GRAPPA, Science Park 904, 1098, XH, \\ Netherlands; "NASA-MSFC USA
}

\begin{abstract}
The calibration system for XIPE is aimed at providing a way to check and correct possible variations of performance of the Gas Pixel Detector during the three years of operation in orbit (plus two years of possible extended operation), while facilitating the observation of the celestial sources. This will be performed by using a filter wheel with a large heritage having a set of positions for the calibration and the observation systems. In particular, it will allow for correcting possible gain variation, for measuring the modulation factor using a polarized source, for removing non interesting bright sources in the field of view and for observing very bright celestial sources. The on-board calibration system is composed of three filter wheels, one for each detector and it is expected to operate for a small number of times during the year. Moreover, since it operates once at a time, within the observation mode, it allows for simultaneous calibration and acquisition from celestial sources on different detectors. In this paper we present the scope and the requirements of the on-board calibration system, its design, and a description of its possible use in space.
\end{abstract}

Keywords: X-ray polarimetry

\section{INTRODUCTION}

50 years after the first pioneering experiments, X-ray observations are a well-established tool in Astronomy. Enormous progress in instrumentation has allowed for highly performing imaging, spectroscopic and timing experiments. Only one observing technique is still basically missing - polarimetry. XIPE, the X-ray Imaging Polarimetry Explorer, is a mission concept selected by ESA as a M4 candidate (with a possible launch in 2026), aimed to measure $\mathrm{X}$-ray polarization from celestial sources.

Polarimetry adds two more observables, in addition to the position, energy and arrival time of each photon: the degree and angle of polarization. The former gives direct insight in to the emission mechanism and the geometry of the source, while the latter may often provide the only way to measure the orientation of the system. This will allow one to test Quantum electro-dynamics (QED) and general relativity (GR) effects and to investigate particle

Space Telescopes and Instrumentation 2016: Ultraviolet to Gamma Ray, edited by

Jan-Willem A. den Herder, Tadayuki Takahashi, Marshall Bautz, Proc. of SPIE Vol. 9905, 99054H

- (c) 2016 SPIE · CCC code: 0277-786X/16/\$18 - doi: 10.1117/12.2233448

Proc. of SPIE Vol. $990599054 \mathrm{H}-1$ 
acceleration mechanisms, past activity and aspherical geometries for a number of astrophysical sources at all scales, ranging from white dwarfs and pulsars, pulsar wind nebulae and supernova remnants, binaries, molecular clouds in the Galactic Center, to distant AGNs. Polarimetry effects are also predicted by the Loop Quantum Gravity and can be tested by observing distant polarized sources like blazars.

The XIPE instrument design takes advantage of previous studies, and in particular: (i) the assessment study of XEUS/IXO by ESA/NASA/JAXA; (ii) the successful phase A study of POLARIX, a mission proposed in response to a call by the Italian Space Agency (ASI; (iii) the study of NHXM, a mission proposed to ESA M3 call. The XIPE payload (see a separate paper in these proceedings for all details ${ }^{1}$ ) is basically divided in three main parts; the Mirror Assembly (MA), the Focal Plane Assembly (FPA) and the Instrument Control Unit (ICU). The FPA assembly is the main responsibility of the XIPE consortium, and is based on three identical photoelectric X-ray polarimeters exploiting the newly developed Gas Pixel Detector (GPD) design ${ }^{2,3,4}$. The key characteristic of the GPD is that it is capable to perform imaging polarimetry, time and energy resolved, with adequate sensitivity for astronomical observation. This innovative technology, which is based on the photoelectric effect, foresees the use of a gas cell for converting photons in photoelectrons, an electric field for drifting the photoelectron track towards the multiplication stage, a gas electron multiplier to amplify the charges of the track, and, eventually, an ASIC CMOS multilayer chip with hexagonally pixelated top layer for charge collection while the bottom layers are the front-end electronics for a complete analog signal processing. The second cutting edge technology foreseen on board XIPE is the thin-shell technology, already successfully employed in the eRosita experiment on-board the Spectrum X-Gamma mission. This technique will be employed for developing the shells of the XIPE X-ray telescopes with Iridium and Carbon coating, enabling good quality mirrors with a relatively low weight and enhanced reflectivity.

The focal plane assembly of XIPE will host three identical GPDs, each hosted in a different Detection Set (DS); each DS will contain the components necessary to operate and calibrate the detector:

- $\quad$ a GPD (the detector)

- a Filter Wheel (FW)

- $\quad$ a baffle, which shields the detector from the diffuse X-ray photons not coming from the observed cosmic source

- $\quad$ a Back End Electronics (BEE), which power and manage the Detector Unit (DU).

The schematic design of the telescope is illustrated in Figure 1. Two out of the three X-ray telescopes are shown, with the optics on the left, illuminating the detection unit (DU), which contains the filter wheel and the Gas Pixel Detector GPD. Next to the DU there is the back end electronics (BEE) unit.

For all details of the payload we refer to a separate paper in these proceedings ${ }^{1}$. In this paper we concentrate on one of the key components of the XIPE payload and focal plane, i.e. the set of filter wheels that will allow to position on board

calibration sources and filters in front of the detector. Their design and requirements are illustrated in the following sections; FW design and provisions are supported by a large heritage from past missions and are led by MSSL-UCL.

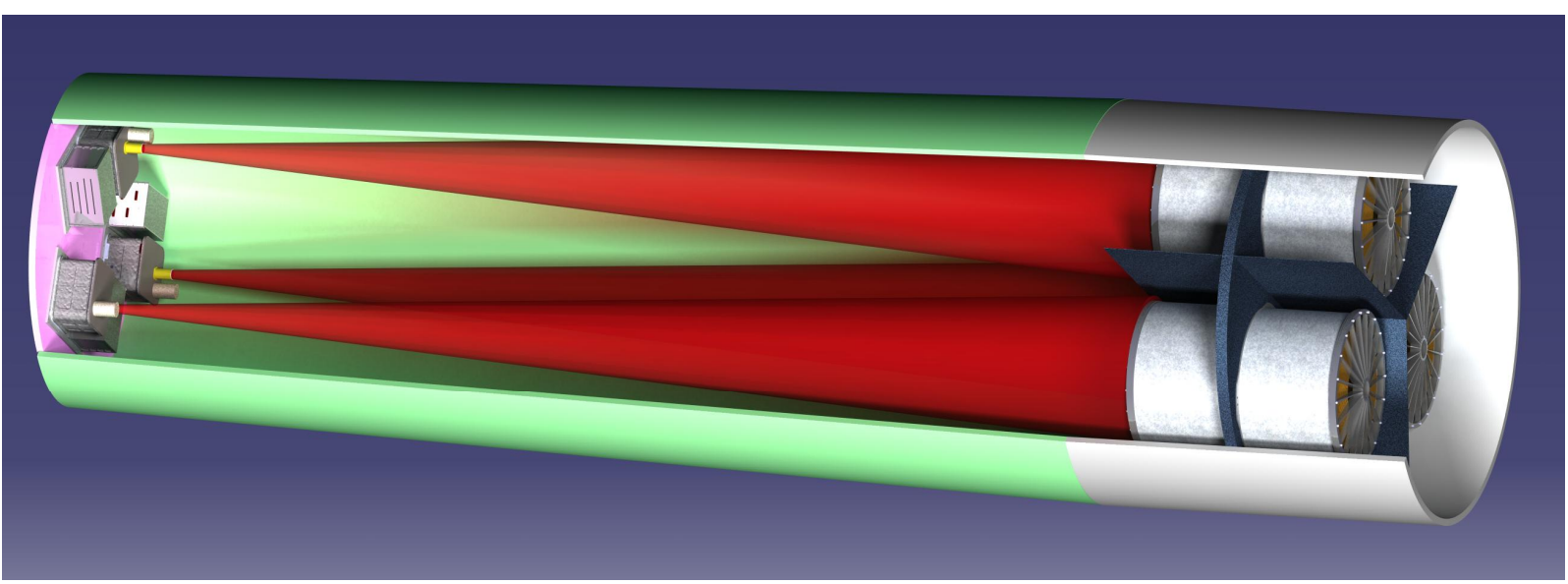

Figure 1. An outline of the XIPE telescope. See a separate paper in these proceedings for all details ${ }^{1}$. 
Figure 1 shows an outline of the telescope, without the spacecraft. On the left the 3 focal plane GPD units with back end electronic boxes and on the right the $3 \mathrm{X}$-ray mirror assemblies, separated with a (cut open, green) CFRP telescope tube. The focused X-ray beams are shown as translucent red cones, from the focusing optics to the detector entrance baffle. The XIPE consortium is responsible for the overall design of the detector units with support electronics, including an instrument control unit (not shown) and the CFRP sandwich support panel (disc shape in pink).

\section{FILTERS AND CALIBRATION SOURCES REQUIREMENTS}

The purpose of the filter wheel is to provide for various apertures which can be chosen using the Instrument Control Unit. In this paper we describe the baseline configuration, in which each filter wheel will have 8 positions; the requirement of a further position, with an additional filter for bright sources $(>2 \mathrm{Crab})$ is under discussion in the XIPE science team and eventually will require only minor modifications to the design. In the current baseline design, the 8 positions of the FW are the following:

1. Open position. In this position no filter is put in front of the detector. The requirement on the minimum clearance $d$ of the aperture can be calculated by the formula (see Figure 2)

$d>\left[15 \sqrt{2}+\frac{L}{F}(D-15 \sqrt{2})\right] 1.15 \mathrm{~mm}$

Where $\mathrm{L}$ is the distance between the focal plane (corresponding to the middle of the GPD gas cell) and the filter wheel plane; F and Dare the focal length and the diameter of the XIPE telescope, respectively. The factor $15 \sqrt{2}$ is the GPD ASIC size, while the factor 1.15 accounts for a $15 \%$ margin. Assuming the baseline configuration values for all of these parameters, i.e. $L=65 \mathrm{~mm}, \mathrm{~F}=4000 \mathrm{~mm}, \mathrm{D}=400 \mathrm{~mm}$, gives a constraint on the aperture value of $d>31.8 \mathrm{~mm}$.

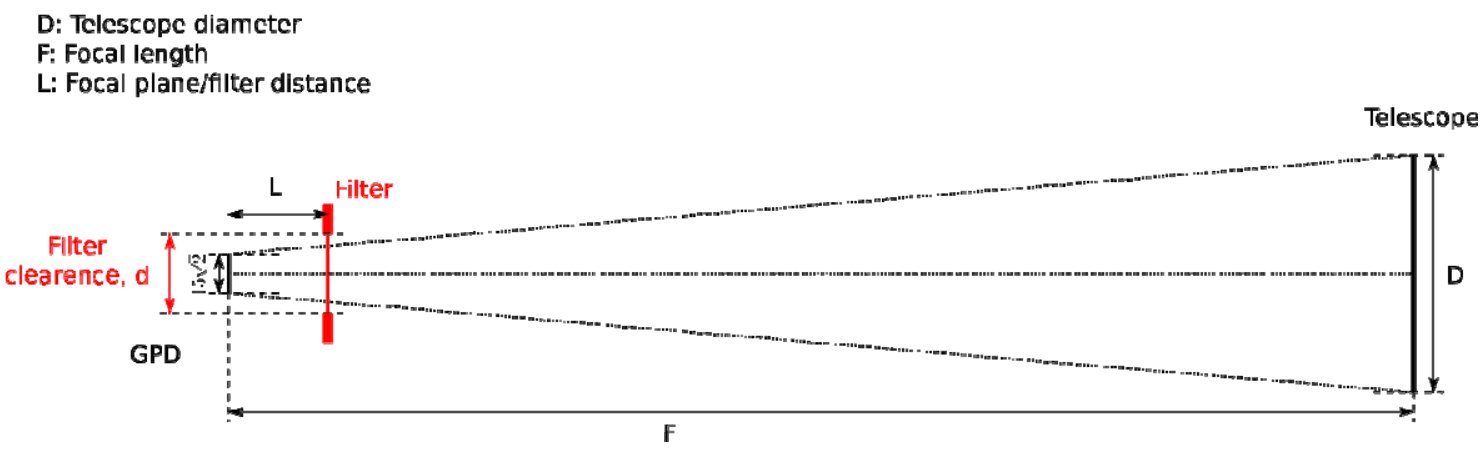

Figure 2. Aperture definition.

2. Close position. In this position a black (opaque) filter, is placed in front of the detector. As a baseline, the filter will be a disk of tungsten $0.5 \mathrm{~mm}$ thick, with a multilayer coating of different materials with lower atomic number to absorb the tungsten fluorescence lines. The diameter of the filter can be calculated with the same formula used above to derive the clearance of the open position. Assuming a conservative diameter of $40 \mathrm{~mm}$ and allowing $2.5 \mathrm{~mm}$ to hold the filter in place, the diameter of the filter will be $45 \mathrm{~mm}$. The corresponding weight is $\sim 15 \mathrm{~g}$.

3. Grey filter. In this position a filter which is partially opaque to the radiation of interest is used. As a baseline, the filter will be a disk of the same size as the black filter used in the close position, but made of Beryllium and $0.25 \mathrm{~mm}$ thick; in this case, the weight is $\sim 1 \mathrm{~g}$.

4. Diaphragm. A tungsten diaphragm, $0.5 \mathrm{~mm}$ thick, is placed in front of the GPD. The diaphragm will have the same size as the grey and the black filters, with a hole in the centre of $4 \mathrm{~mm}$.

5. Calibration source A. A source of polarized photons with a ${ }^{55} \mathrm{Fe}$ radioactive source (see Muleri et al. 2007 Proc. of SPIE 6686:668610). The preliminary drawing is reported in Figure 3. A larger laboratory version made of aluminium (without the radioactive source) of size $47 \times 47 \times 50 \mathrm{~mm}^{3}$ weights about $150 \mathrm{~g}$; we assume for the flight version a comparable/lower mass. Pictures of the laboratory version are shown in Figure 4. 
The bottom of the source (see Figure 3) should be as close as possible to the GPD to increase the number of photons collected, with a minimum distance of $15 \mathrm{~mm}$ (see Figure 5). Alternative designs for this source will be evaluated during the XIPE definition phase. Is important to notice that designs in which the source is active (i.e. it has to be powered) require that its position is away from the movable part of the filter wheel.

6. Calibration source B (collimated unpolarized source). This source will be composed of a ${ }^{55} \mathrm{Fe}$ iron radioactive source and two $1.7 \mathrm{~mm}$ diaphragms $30 \mathrm{~mm}$ apart. The source will be hosted in a hollow cylinder with height lower than the maximum height of the calibration source A (which is about $40 \mathrm{~mm}$, see Figure 5) and diameter $2 \mathrm{~cm}$. The cylinder and the diaphragms can be made of brass, $1 \mathrm{~mm}$ thick, in which case the weight of the source is $\sim 20 \mathrm{~g}$. The lower diaphragm should be placed as close as possible (with a minimum distance of $15 \mathrm{~mm}$ ) to the GPD top frame to reduce the spot size on the detector.

7. Calibration source $\mathbf{C}$ (isotropic unpolarized source \#1). This source will be composed of a ${ }^{55} \mathrm{Fe}$ iron radioactive source, with its holder made of brass. The volume will be roughly cubic, of the order of $1000 \mathrm{~mm}^{3}$, and the weight is $\sim 8 \mathrm{~g}$.

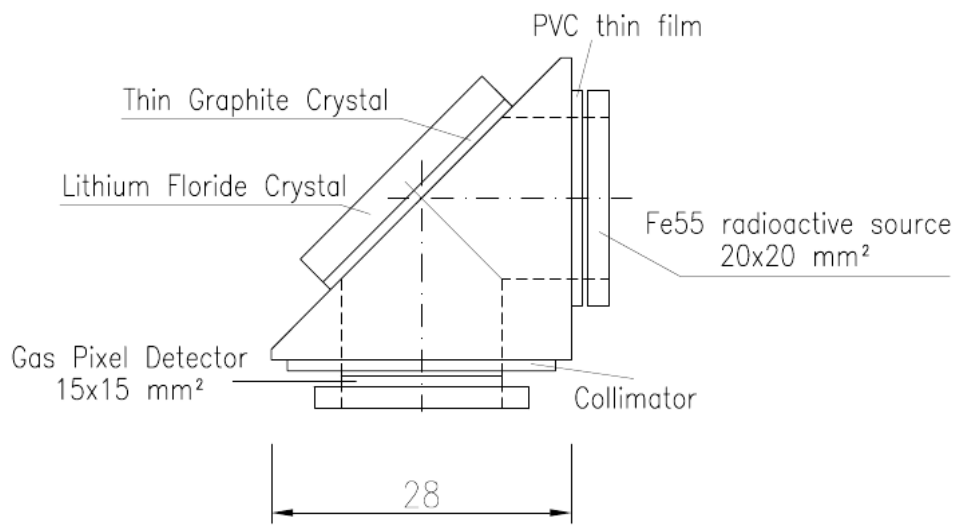

Figure 3. Schematic drawing of the polarized source
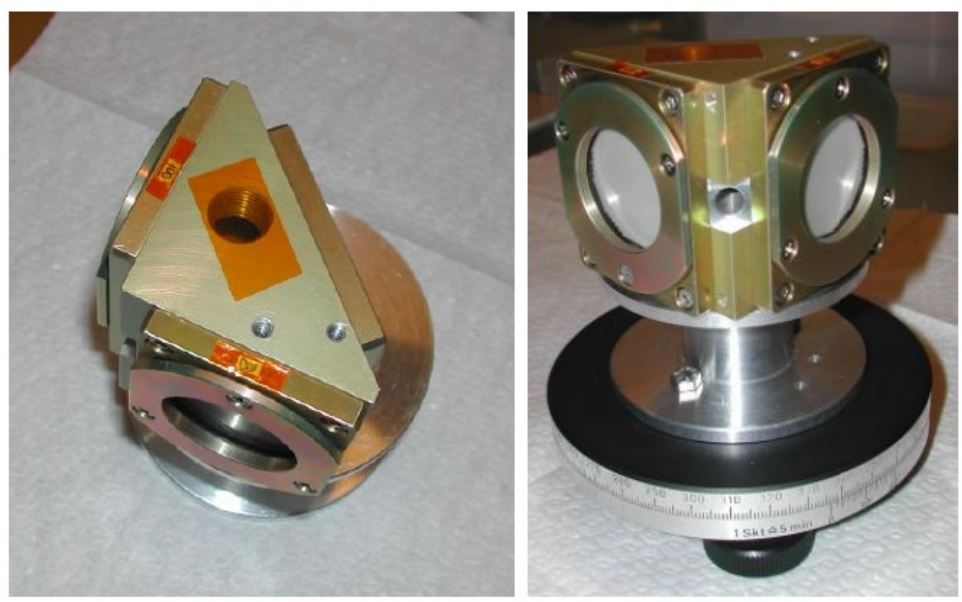

Figure 4. Laboratory model of the polarized source.

8. Calibration source $\mathbf{D}$ (isotropic unpolarized source \#2). This source will be composed of $\mathrm{a}^{109} \mathrm{Cd}$ radioactive source in its holder made of copper, which is also used as fluorescence target. The volume will be of the order of (length $\mathrm{x}$ width $\mathrm{x}$ height) $15 \times 15 \times 20 \mathrm{~mm}^{3}$, and the weight is $\sim 40 \mathrm{~g}$. 
Depending on the filter wheel motor mounting, the filters/sources can be placed above or below the filter wheel plane; however, care should be paid when reducing the distance between the filters and the GPD window. In fact, the minimum distance between any part of the FW and the detector/focal plane must be $15 \mathrm{~mm} / 25 \mathrm{~mm}$ due to the presence of high voltage $(-3.5 \mathrm{kV}$ maximum; see Figure 5). The mechanical interface between the filters and calibration sources and the FW will be identical for all of the positions.

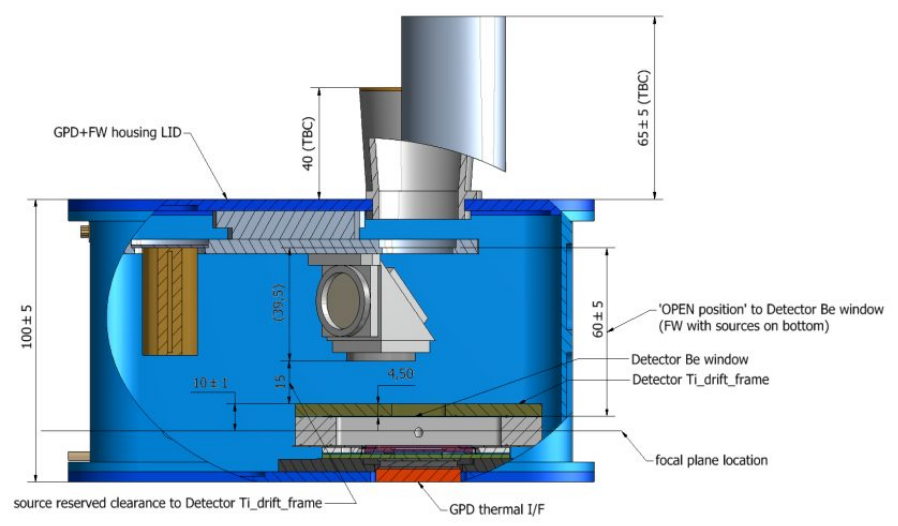

Figure 5. Constraints on the distance between the FW and the detector/focal plane

\subsection{Positioning requirements}

The accuracy and repeatability of the positioning of the filter wheel in each position are the following:

1. Open/close/grey filter positions: no requirement on the positioning/repeatability (other than the clearance).

2. Diaphragm: accuracy $<0.35 \mathrm{~mm}$ and repeatability $<0.35 \mathrm{~mm}$. These requirements are set to provide a negligible jitter $(<0.5 \mathrm{arcmin})$ on the field of view of the instrument, while the diaphragm is used.

3. Calibration source $\mathbf{A}$ : the accuracy on the angular positioning of the wheel $<3.5 \operatorname{arcmin}$ (see Figure 6); and repeatability $<3.5$ arcmin. These requirements are particularly important to ensure the stability on the measurement of the angle of polarization: the science requirement on the knowledge on the polarization angle with respect to the sky is 5 arcmin.

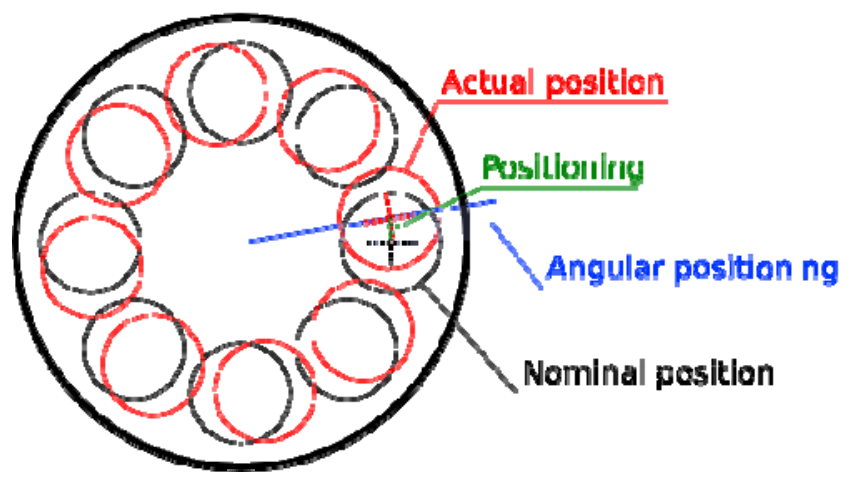

Figure 6. Illustration of the definition of angular positioning for the FW.

4. Calibration source $\mathbf{B}$, accuracy $<1 \mathrm{~mm}$ and repeatability $<1 \mathrm{~mm}$. These requirements are set to check consistently the same region of the detector (the spot will be about $3 \mathrm{~mm}$ large).

5. Calibration source $\mathbf{C}$ and $\mathbf{D}$, accuracy $<0.5 \mathrm{~mm}$ and repeatability $<0.5 \mathrm{~mm}$. These requirements are set to illuminate the whole detector surface with an axially-symmetric distribution of photons. 
In addition, the parallelism between the filter wheel plane and the focal plane should be better than 0.1 deg to ensure that the modulation curve measured with polarized and unpolarized calibration sources does not systematically deviates from the expected cosine square dependency.

\subsection{Use of the filter wheel and radiation harness}

The lifetime of the XIPE mission is 3 years, plus 2 additional years of extended operations. During this period, the filter wheel is expected to be operated sporadically. More specifically, the open position will be the standard position of the filter wheel during most of the scientific observations, while the closed position will be used at launch and for measuring the internal background, with a frequency of twice a year. Both grey filter and diaphragm are expected to be used $<10$ times a year, while the frequency of use will be $\sim 1$ month for calibration source $\mathrm{A}, \sim 3$ months for calibration sources B and C and 6 months for calibration source D. Therefore, each filter wheel will be required to be moved about 50 times a year.

A first estimate of the radiation dose after 5 years in orbit for the elements inside the Detection Unit (where the FW will be placed) is about $200 \mathrm{rad}$.

\section{FW CONCEPT: TRADE OFF STUDIES}

The design of the FW is determined by the trades below:

- Orientation of the wheel with regard to the gas pixel detector, facing towards or away.

- Distance between filter wheel and high voltage gas pixel detector also considering the aperture size.

- Mechanism transients, considering the time it takes to move from one filter position to the next which defines the driving torque requirement.

- $\quad$ Reliability, taking into account the ESA requirements.

- Rotational accuracy and repeatability.

- Position sensors, encoders, fail safe, redundancy.

- Electronics Block Diagram, considering full redundancy.

- Mass budget although not critical as such impact of design trades needs to be traced.

- $\quad$ Filter wheel balance, primarily to minimise mechanical disturbance in orbit and avoid additional loads during ground testing.

We will discuss the most important trades in detail in the following subsections.

\subsection{Orientation}

The filter wheel will hold several sources, and these sources have an envelope that protrudes significantly away from the FW itself. It is therefore important to decide the relative orientation (see Figure 7). Pointing the sources enclosures away from the GPD requires a smaller aperture opening and therefore a smaller filter wheel and less mass (Figure 8). However, pointing upwards makes the mounting of the wheel difficult with regard to the lid of the enclosure. Since the lid will hold the entrance baffle for the GPD, the FW would have to be mounted either next or on top of the GPD or at the end of a structure lowering the filter wheel from the top, negating the mass savings. As a result, the stepper motor will be located inside the enclosure, including the required harness. This may impact the thermal stability of the GPD, and may require further design to ensure that no harness will run through the enclosure near the filter wheel. None of the above arguments are in themselves show stoppers. However, in absence of strong motivations against, it appeared more sensible to adopt as a baseline the configuration inherited from previous studies, with a pointing towards the GPD (the current design leaves a $65 \mathrm{~mm}$ gap between the wheel and the GPD). This allows for mounting of the filter wheel on the lid of the enclosure with the stepper motor and associated harness outside the enclosure; the heat generated by the stepper motor is then dissipated outside the enclosure.

The baseline allows for a single sub structure that holds the filter wheel and the entrance baffle and, at the same time, serves as a lid for the enclosure. The harnesses (apart from those associated with the location sensors) are routed outside the enclosure. This baseline appears therefore as a self-contained assembly.

There is an additional requirement now to have access to some of the calibration sources prior to launch that needs to be addressed in the near future. In fact some of the sources are radioactive and become weaker after few years, therefore they need replacing shortly before launch to ensure the maximum life in orbit. 


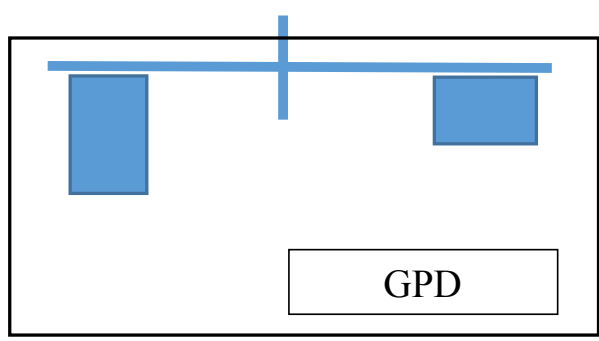

Baseline

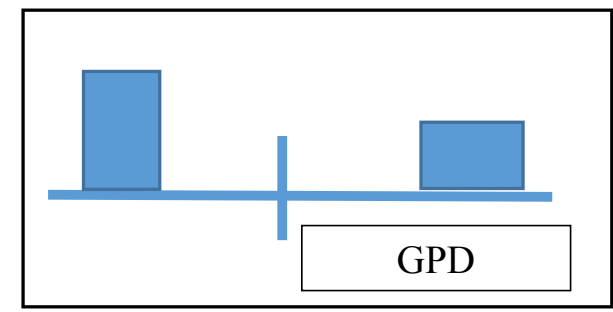

Alternative with smaller aperture

Figure 7 Filter wheel orientation

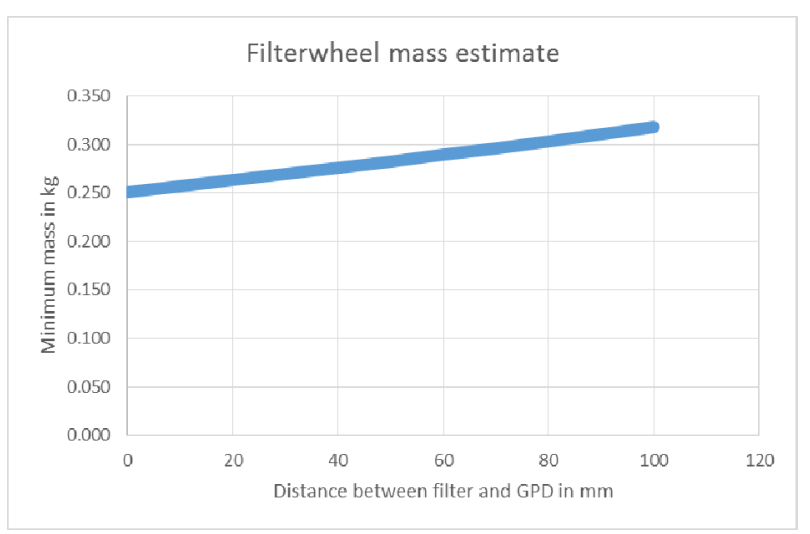

Figure 8 Filter wheel mass sensitivity

\subsection{Distance between FW and GPD and aperture size}

As discussed, in the baseline, the FW mounted components are pointing towards the GPD. Due to the high voltage component of the GPD itself, there is a minimum required distance between these components and the GPD. In turn, the distance between the FW apertures and the optical plane within the GPD determines the minimum aperture size required. The distance to the optical plane inside the GPD, i.e. the plane where the X-rays are focused, is $10 \mathrm{~mm}$ from the front surface; the minimum required clearance between the top surface of the GPD and components mounted on the filter wheel is $15 \mathrm{~mm}$ (see Figure 9).

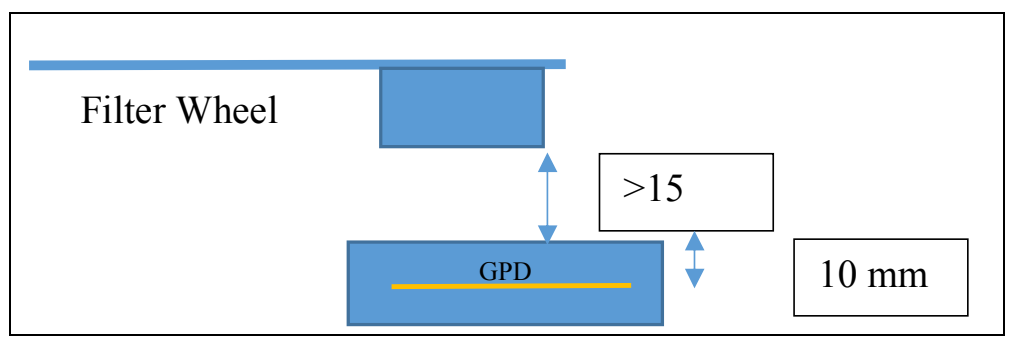

Figure 9: Minimum distance to the component from top surface, and distance between the top surface and the optical plane inside the detector.

As illustrated in Figure 9, the distance between the FW and the GPD is determined by the tallest component mounted on the FW and by the minimum clearance required between that component and the GPD (i.e. the minimum distance needed not to cause high voltage breakdown), plus an additional $10 \mathrm{~mm}$ to the optical plane within the detector. This defines effectively the minimum required aperture in the filter wheel.

The tallest component at present is calibration source B. Allowing for $15 \mathrm{~mm}$ space between the high voltage surface of the GPD and the components on the filter wheel we need a separation of $65 \mathrm{~mm}$ between the GPD focal plane and the filter wheel. With a GPD diameter of $21.21 \mathrm{~mm}\left(15 \times 15 \mathrm{~mm}^{2}\right)$ this yields an aperture of $32.5 \mathrm{~mm}$ including $15 \%$ margin. 


\subsection{Filter wheel movement transients}

The most important factor that defines the power required to move the FW is the requirement for the time to move the filter wheel from one position to the next one. The movement of the FW is characterized by three stages: a first phase is ramp up, a second phase is constant speed and a third phase is ramp down. Ideally this must be achieved when moving to the next filter position, since in this case the stepping from filter to filter will only depend on the length of time spent during the constant speed phase: this simplifies the control of the FW.

The rotational inertia and the time to speed up or down are the main factors define the driving torque requirements. For the following analysis we assumed a filter wheel with a diameter of $85 \mathrm{~mm}$ to be conservative. The rotational inertia is $0.001667 \mathrm{~kg} / \mathrm{m}^{2}$. Assuming a time of $2 \mathrm{~s}$ to move to the next position, a full rotation would then take $16 \mathrm{~s}$ minus 7 times the ramp up time (ignoring friction effects and shock loads as the stepper motor rotates). Figure 10 shows the different speed profiles required to drive the filter wheel to the next position within $2 \mathrm{~s}$ : as the effective rotation is $2 \pi / 8$, the areas under each graph have to be equal. As can be seen from the speed profile and the corresponding torque profile. The obvious conclusion is that driving the filter wheel at a lower speed requires less torque. When a mechanism is tested, and we are planning to do a life test model (LTM), the required safety factors are lowered. The present design takes into account for these lower safety factors. For the purpose of the design trade we assume that the worst case torque of $34 \mathrm{mNm}$ is required, this covers all the required margin.

At present there are no driving requirements for the duration of the transition time allowed to move from one position to the next, but, as a general rule, it is better to drive the wheel slowly and in the same direction each time. The mechanical disturbance is then minimised and the life of the gears extended.

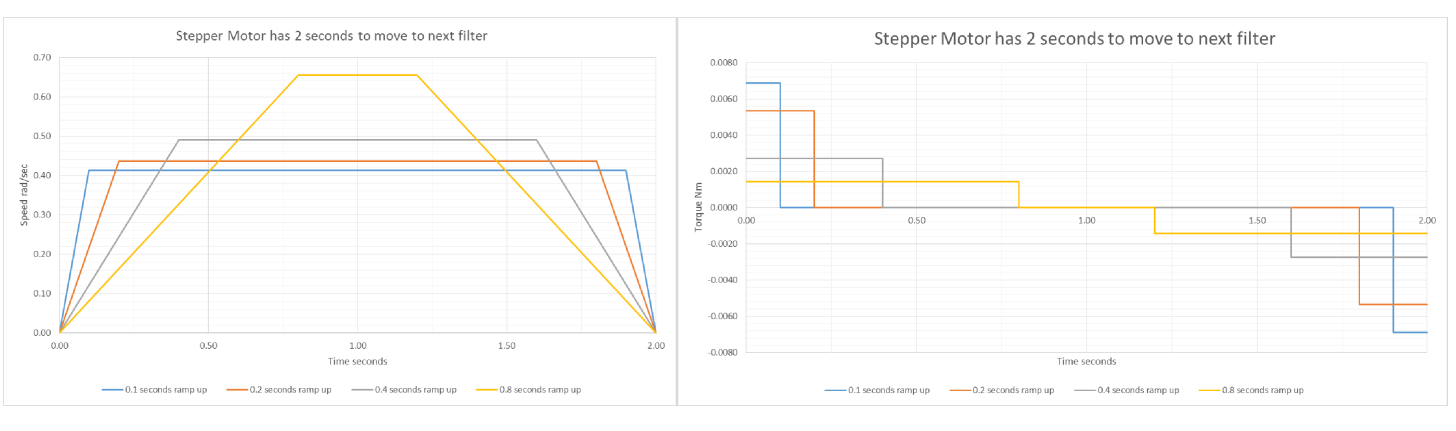

Figure 10: Filter wheel speed and torque profile

The profiles shown in figure 10 are for sizing the stepper motor from first principles, the speed profile using an actual stepper motor will look different, but figure 10 is sufficient to size the required torque for the stepper motor.

\subsection{Reliability, Mechanical, Thermal and Electronics}

The reliability of the FW impacts on thermal, mechanical and electrical aspects. The stepper motor needs to be powerful enough to drive the FW, and since the thermal environment of the FW for XIPE is room temperature throughout the mission we do not expect exposures to temperature extremes that could affect the bearings or the electronics adversely. The mechanism needs to be able to sustain a bake out and some margin on the operating temperatures. The cold survival case has not been defined yet, but, since the GPD needs to be protected at all times and will be controlled closely around $17^{\circ} \mathrm{C}$, is not expected to be severe. Currently, the operational temperature range is assumed to vary between $10^{\circ} \mathrm{C}$ and $30^{\circ} \mathrm{C}$, a cold survival is set as provisional at $-20^{\circ} \mathrm{C}$, and bake out is set at $+80^{\circ} \mathrm{C}$. Overall, the thermal environment at present is not considered a significant risk.

The stepper motor needs to be able to generate enough torque under all operating conditions. This means it needs to be able to generate more torque than required under conditions where the performance is already reduced. Specifically, with regard to reliability, the stepper motor should be able to drive the filter wheel with half the power and twice the inertia. That means the driving torque should be at least 4 times higher than the resisting torque of bearings and other items. We have sized the stepper motor to have at least a factor of 6 torque margin during the phase-A study. It should be noted that this margin is also related to the time allowed to rotate the filter wheel as it is obvious from what discussed in the previous sections.

Another important consideration regards the prime and redundant coils to step the motor. The Faulhaber stepper motors do not have prime and redundant coils, while they are available on the Phytron stepped motors, which also 
have a considerable space heritage. Since the stepper motors are only required to perform a minimal number of operations during the flight and ground testing lifetime (less than 5000 movements), the need for a prime and redundant motor is not strong. However, it appears feasible to foresee the prime and redundant coils as part of a complete redundant system. This redundancy is traded as part of the electronics design (see next sections).

\subsection{Rotational Accuracy and repeatability}

As for the FW design, the most stringent requirement derives from the fact that one of the sources (the source of polarized light) needs to be positioned with a knowledge better than 3.5 arcmin. As a consequence, the FW needs to be capable to locate it within 3.5 arcmin of absolute repeatability.

The initial baseline design was a filter wheel with teeth on the outer edge and with a pinion driven by a stepper motor. The wheel had 180 teeth on its circumference and 12 on the pinion. This gives a drive ratio of 1:15. The 3.5 arcmin absolute location can be translated into a radial displacement on the circumference of the filter wheel itself and this should feed into the backlash budget. For a filter wheel with an effective radius of $94 \mathrm{~mm}$, which allows for an entrance aperture of $39 \mathrm{~mm}$, the 3.5 arcmin position location translates into $0.1 \mathrm{~mm}$. By allowing 50 micron backlash, only leaves 1.75 arcmin uncertainty in the budget.

\subsubsection{Faulhaber option (stepper motor with high TRL)}

Faulhaber stepper motors have a drive ratio 1:15 (meaning that 15 revolutions of the pinion result in 1 revolution of the FW) and 24 steps per revolution of the pinion, resulting in a total of $24 \times 15=360$ steps for the whole filter wheel. The angular uncertainty per step is $15^{\circ}$. The requirement for the angular repeatability of the polarised light source is 3.5 arcmin. With a step uncertainty of $15^{\circ}$ for the stepper motor and a gear ratio of 1:15 this is clearly not achievable. This configuration will in fact give a position uncertainty of $+/-1^{\circ}$, based on the gear ratio and pinion angular position uncertainty.

\subsubsection{Phytron option (Stepper motor with high TRL)}

Phytron has several space qualified stepper motors with enough torque to be suitable for application to XIPE. The stepper motors have 200 steps per revolution of the pinion, which gives 3000 steps for each rotation of the filter wheel. This means that each step corresponds on average to 7.2 arcmin (not taking into account backlash). At present, however, the angular uncertainty is $1.8^{\circ}$ which again is insufficient to match the requirement of 3.5 arcmin.

\subsubsection{Worm wheel design (Using either stepper motor suggested before)}

If the filter wheel is driven using a stepper motor rotating a worm wheel, one full rotation of the stepper motor will move the filter wheel by 1 tooth. Worm wheels can be designed to be nearly backlash free. In principle, by using 24 steps and 600 teeth each step advances the filter wheel by 1.5 arcmin leaving an allowance of 50 microns of backlash. Using a Phytron stepper motor would further reduce the need for 600 teeth to 295 .

\subsubsection{Piezo rotary stage (Different actuator type)}

Another possibility is to use a piezo rotary stage. Piezo actuators currently exist with a holding torque of $6 \mathrm{mNm}$ and an actuating torque of $5 \mathrm{mNm}$ and with a dimension in the range of $18-22 \mathrm{~mm}$. The rotational step resolution is around $1 / 3$ of an arcmin. The piezo rotary stage may be a direct drive on the filter wheel axis but would not be able to hold the mass of the filter wheel. This type of actuator is high-vacuum compatible however, their space heritage at this stage is unknown.

\subsubsection{End Stop}

A simple method to achieve a repeatable angular position for the calibration source is to push the filter wheel into an end stop. This can be done for one or two sources and would require the filter wheel to be driven backwards and forwards. Since the minimum requirements on the step size would still hold, the use of an end stop should be seen as an advantage in terms of repeatability and absolute location, and not necessarily in terms of accuracy which will still depend on the way the wheel will be driven. 
The different options are summarized in Table 1.

\begin{tabular}{|l|l|l|l|}
\hline Option & $\begin{array}{l}\text { Meets the 3.5 } \\
\text { arcmin } \\
\text { requirement? }\end{array}$ & $\begin{array}{l}\text { Minimum time for one } \\
\text { revolution of filter wheel }\end{array}$ & $\begin{array}{l}\text { Absolute location } \\
\text { included }\end{array}$ \\
\hline Faulhaber wheel/pinion & No & $16 \mathrm{sec}$ & No \\
\hline Phytron wheel/pinion & No & $16 \mathrm{sec}$ & No \\
\hline Worm wheel & Yes & $60-120 \mathrm{sec}$ & No \\
\hline Piezo rotation & Yes & $6 \mathrm{sec}^{1^{*}}$ & Yes \\
\hline
\end{tabular}

Table 1. Summary of the options for the filter wheel driver design.

$1 *$ With a commercial controller

The requirement on the repeatability of the location of the filter wheel within 3.5 arcmin implies the need to consider either a worm wheel or a piezo rotation. The worm wheel is slower than all the other methods, however it appears as the most promising solution since it has more heritage than a piezo rotation and the time it takes to rotate one full revolution is not a strict requirement for XIPE.

\section{FW DESIGN}

As discussed in the previous sections, the overarching characteristic for the filter wheel is that it has to hold polarised light sources for the GPD apart from attenuation filters and non-polarised sources. The filter wheel itself is driven using a stepper motor; the polarised source requires angular repeatability for the filter wheel in excess of what has been achieved on previous space missions. A specific design has been then developed for XIPE.

The filter wheel will be made of aluminium with gear teeth for the drive machined directly into the outside diameter and the gear teeth could have a dry lubricant coating applied if it is deemed necessary. The centre of the filter wheel will be supported on a spindle using two bearings with molybdenum disulphide dry lubricant.

The various filters and calibration sources will have the same interface pattern onto the wheel so they can be mounted easily and swapped around to balance the wheel. Figure 12 shows the basic layout of the filter wheel and its various sources and filters. Details of the various filters and calibration sources have been described in the previous sections. The entrance apertures in the filter wheel have been scaled to not obscure the detector with a margin of $15 \%$ on the radius.

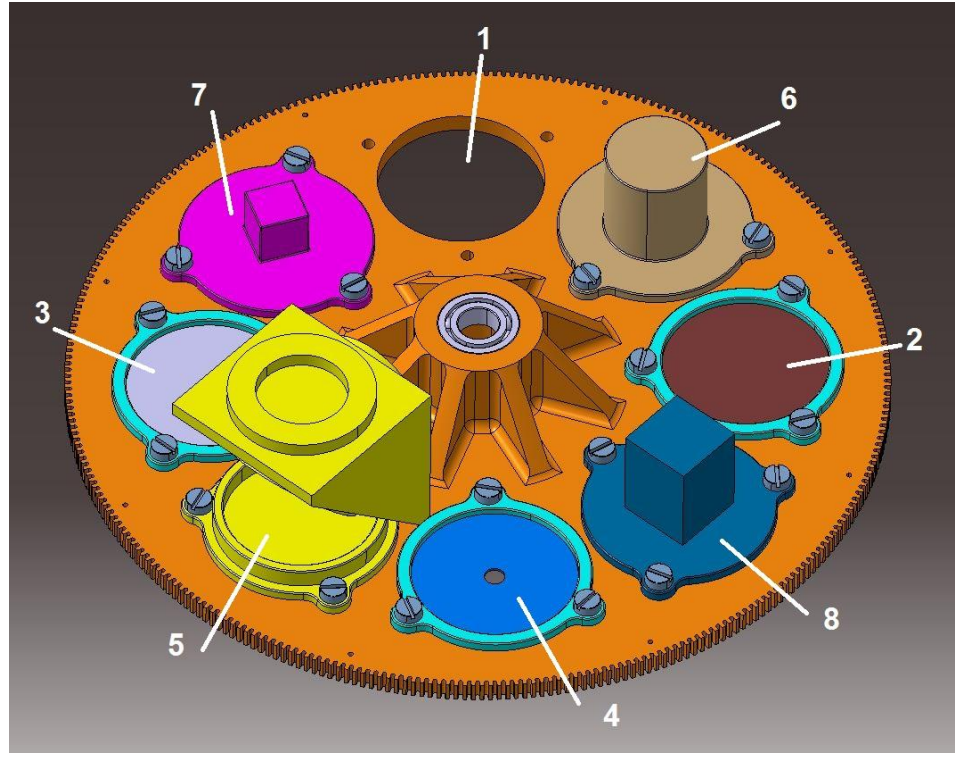

Figure 11. Basic layout of the FW. 1) Open position; 2) Close position; 3) Grey filter; 4) Diaphragm; 5) Calibration source A; 6) Calibration source B; 7) Calibration source C; 8) Calibration source D 


\subsection{Filter wheel mechanism design}

Basing on the trade-off study previously described, the current baseline option for moving the filter wheel is by a stepper motor driving on the periphery of the wheel through a precision ground set of gear teeth. Currently Faulhaber stepper motors as well as Phytron stepper motors are considered.

While a direct drive with a spur gear configuration will at present not meet the fine stepping required for an absolute angular position of the polarised X-ray source, this would be achievable by using a worm wheel gear or a piezo rotary drive. A spur gear arrangement would in fact require an unrealistic gear ratio to meet the fine stepping required for positioning accuracy. In conclusion, after a design trade-off the worm wheel drive, with minimized backlash, was selected as baseline, driven with a stepper motor with built in redundancy (double coils for either prime or redundant power).

The worm gear option gives a significantly higher number of motor rotations for a full filter rotation. With 360 teeth and a space qualified redundant stepper motor (Figure 12) it will be possible to move the filter wheel in steps of subarcmin which will meet the absolute repeatability target. Although absolute position knowledge would likely suffice if it meets the 3.5 arcmin requirement, this would still require fine stepping and a near backlash free movement.

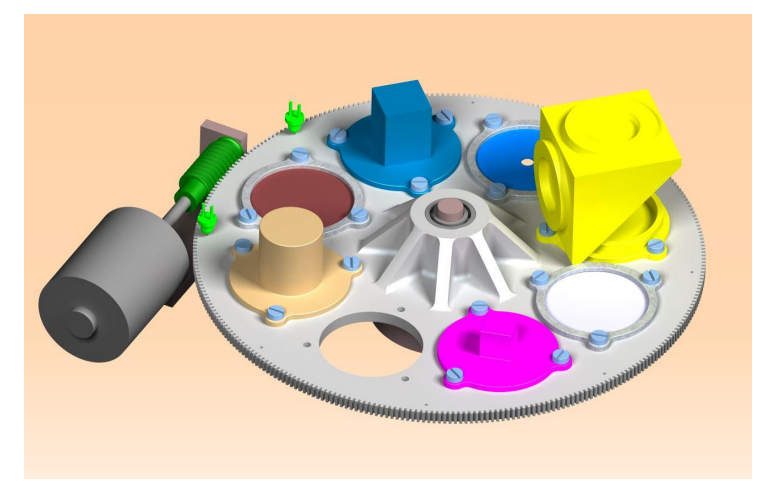

Figure 12. The filter wheel with a worm gear, the baseline configuration which meets the instrument requirements. Figure 12 shows the layout of the filter wheel actuated by a worm wheel with two LEDs indicating where the absolute angular location of filters is measured via pin holes in the filter wheel.

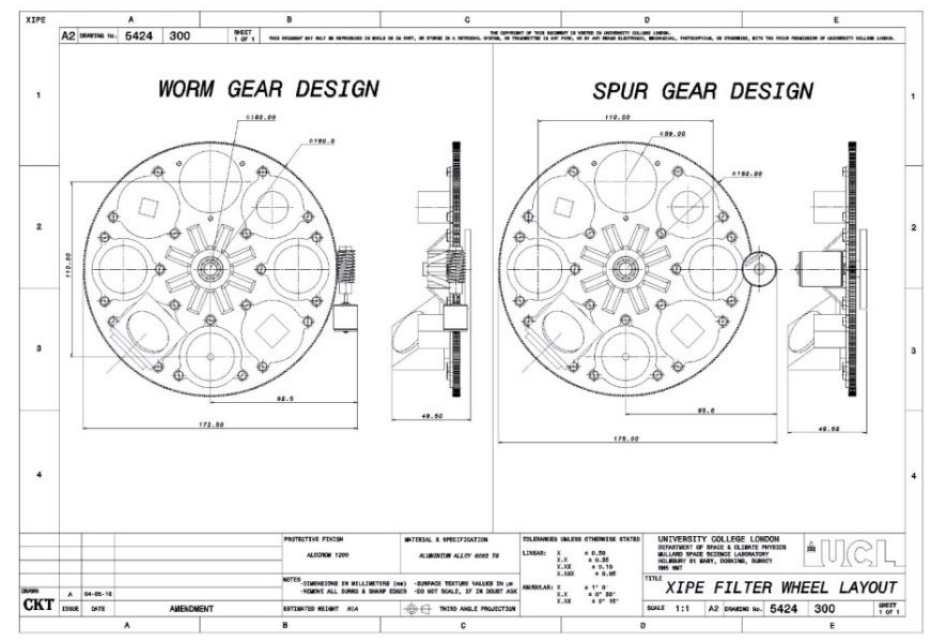

Figure 13. Worm gear and spur gear designs.

As for the choice of the stepped motor is concerned the main point is that, although the Faulhaber motors are small, lightweight and have spaceflight heritage (they are in fact currently used by MSSL for ExoMars), they have only a single winding on the motor poles. This introduces a single point failure in the design. Phytron motors are larger and 
have a mass penalty, but they can be equipped with redundant coils. Therefore, at the moment the baseline is to use Phytron motors to drive the worm wheel, with 200 steps per revolution.

The filter wheel has no need for a specific thermal design as there is no power dissipation on the wheel itself. The stepper motor is firmly attached to the mounting frame where the heat will dissipate. The stepper motor is only active for brief periods of time (order of 30 to 60 seconds) and the power dissipation is expected to be minor.

\subsection{Angular position; optical metrology}

The primary optical metrology for the filter wheel at present is a photo diode quadrant illuminated through a pin hole in the filter wheel using a diode. The principle of this system relies on the balance in illumination between the different sectors of the photo diode which is used to deduce the filter wheel angular position within less than 50 micron. The quadrant can be used to identify a specific location on the filter wheel as the angular positioning of the pinhole only requires two sectors of the quadrant, the other two can be used to distinguish between different locations by varying the radial position of the pin hole for each filter location. The photo diode can be "doubled" up for redundancy and has no effect on the movement of the filter wheel like a mechanically driven optical encoder would.

\subsection{Filter wheel electrical design}

The commanding and control of the three filter wheels is achieved through a dedicated filter wheel control board (FCWB). This board is capable of commanding all three filter wheels and is nominally located within the ICU box. Power and commanding is realised through the ICU backplane.

Cold redundancy is achieved by duplicating the filter wheel control board electronics and can be accommodated on the same card. Two functionally isolated sets of electronics will be populated on the same PCB and ideally communicate with the ICU using different routes though the backplane. A serial protocol will be used for communication. The current baselined position sensing scheme makes use of quadrant photodiodes which will require the amplification and digitisation to be done at FW level

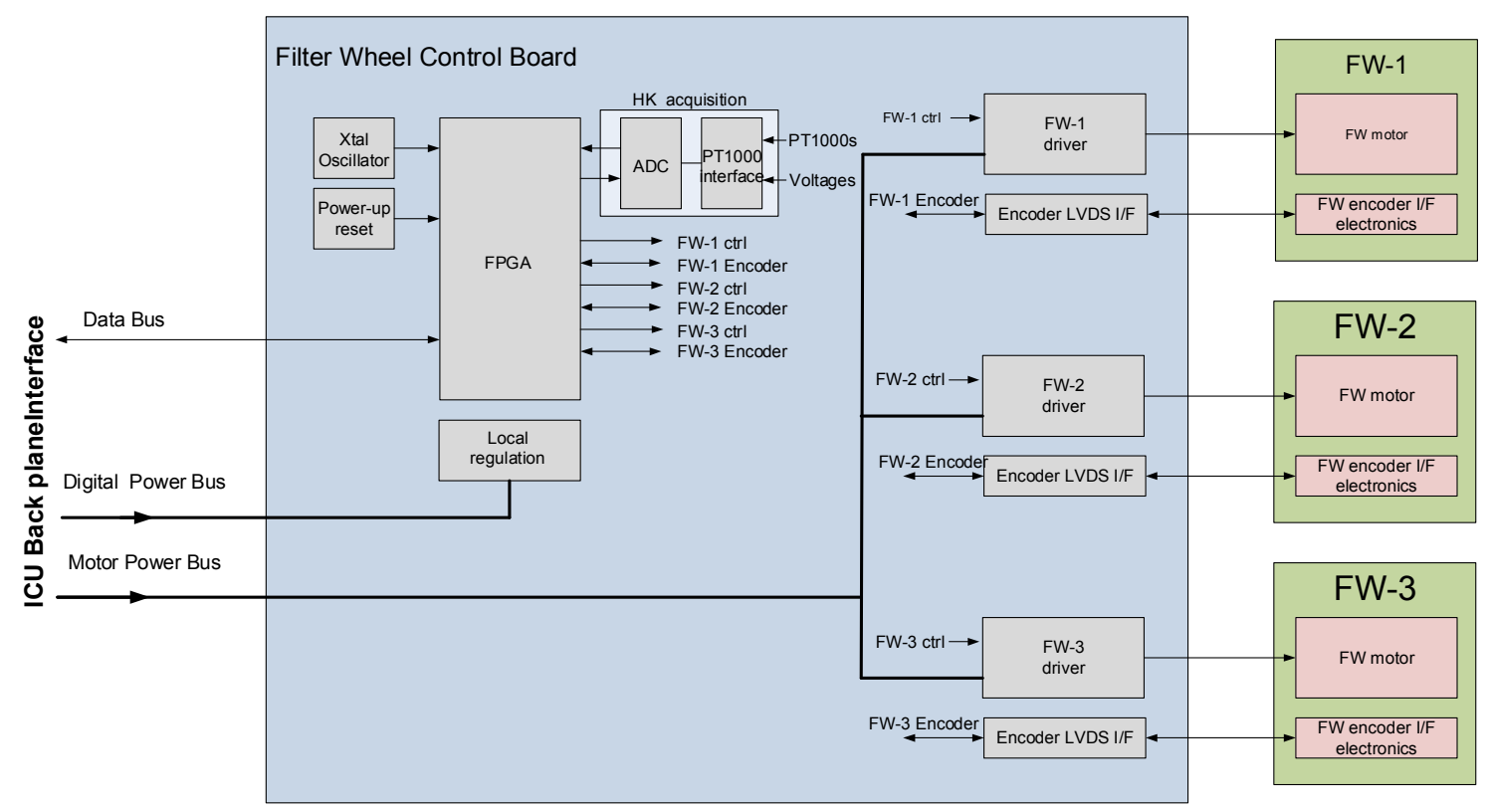

Figure 14. Functional block diagram of the FWCB electronics.

Figure 14 shows the functional block diagram of the FWCB electronics. Each set of electronics independently interfaces with the ICU through the backplane using a serial data bus. The ICU needs to provide two different voltages to the FWCB: a low voltage $(\sim 5 \mathrm{~V})$ regulated digital bus, which will be locally regulated to power the digital electronics, and a higher voltage to power the motors. The motor power bus may be unregulated but will need to be supplied from the secondary side of the DC/DC. 
A single FPGA is used in each set of electronics which is responsible for: communicating with the ICU using a serial bus, interface with the local ADC and provide local telemetry, control the stepper, and interface to the photodiode based position encoders at the FWs. The FPGA will be controlling the stepper motor in a microstepping mode so as to ensure a smother operation of the FW.

Position sensing of the FW will use quadrant photodiodes which entails shining a light source on the photodiode. The light passes through a pinhole arrangement placed near each filter on the filter wheel, which results in a relatively collimated light beam. As the filter wheel rotates, the light bean illuminate's different portions of the quadrant photodiode. When the light distribution between the different quadrants is uniform, within a certain margin, the filter is accurately positioned. Due to the physical separation between the photodiodes and the FWCB, a separate smaller board will be required for each filter wheel. This board will condition the low level analogue signal from the photodiodes and digitise it, so it will be read and compared by the FPGA. The FPGA will store information on the steps counting between the filters and on the coarse location. As the filter wheel approaches the filter, the wheel will slow down until a

separate signal from the encoder board will notify that the light is shining on the first quadrant of the photodiode. Once that signal is received, the FPGA will sample the photodiode voltages and identify when to stop. Communication between the FWCB and the encoder boards will be done using SPI protocol over LVDS.

Each filter wheel will interface to the FWCB with a single harness that will carry both power and data. A redundant harness will be used for the redundant electronics set. The general wiring diagram can be seen in Figure 15; micro-D 25 way connectors are used for this connection. A possible pin arrangement for the 25way MDM connector is illustrated in Table 2, while Table 3 contains a summary of the estimates for mass, power dissipation and dimensions of the different PCBs.

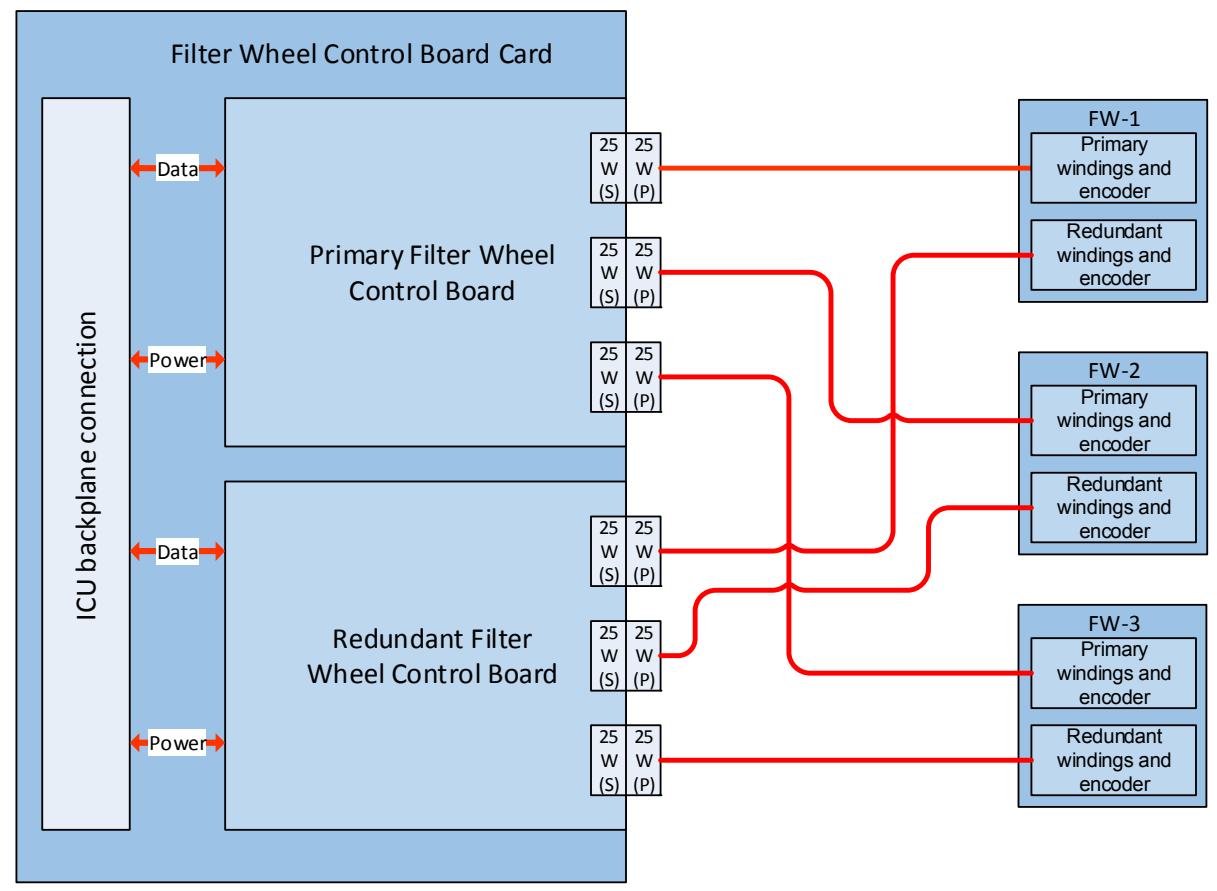

Figure 15. Functional block diagram of the FWCB electronics. 


\begin{tabular}{r|l|r|l}
\multicolumn{1}{r|}{ Pin } & Function & Pin & Function \\
\hline $\mathbf{1}$ & CS_P & $\mathbf{1 4}$ & CS_N \\
\hline $\mathbf{2}$ & MOSI_P & $\mathbf{1 5}$ & MOSI_N \\
\hline $\mathbf{3}$ & MISO_P & $\mathbf{1 6}$ & MISO_P \\
\hline $\mathbf{4}$ & DET_P & $\mathbf{1 7}$ & DET_N \\
\hline $\mathbf{5}$ & DIG_RET & $\mathbf{1 8}$ & DIG_PWR \\
\hline $\mathbf{6}$ & DIG_RET & $\mathbf{1 9}$ & DIG_PWR \\
\hline $\mathbf{7}$ & EMC & $\mathbf{2 0}$ & EMC \\
\hline $\mathbf{8}$ & N/C & $\mathbf{2 1}$ & N/C \\
\hline $\mathbf{9}$ & N/C & $\mathbf{2 2}$ & PH_A \\
\hline $\mathbf{1 0}$ & PH_A & $\mathbf{2 3}$ & PH_B \\
\hline 11 & PH_B & $\mathbf{2 4}$ & PH_C \\
\hline 12 & PH_C & $\mathbf{2 5}$ & PH_D \\
\hline 13 & PH_D & & \\
\hline
\end{tabular}

Table 2

\begin{tabular}{|l|c|c|c|} 
& $\begin{array}{l}\text { Power } \\
\text { (W) }\end{array}$ & $\begin{array}{l}\text { Dimensions } \\
\text { (cm) }\end{array}$ & $\begin{array}{l}\text { Mass per } \\
\text { board (g) }\end{array}$ \\
\hline Motor Control Board - idle & 1.7 & $16 \times 20$ & 140 \\
\hline $\begin{array}{l}\text { Motor Control Board - } \\
\text { motor running (including } \\
\text { motor) }\end{array}$ & 3.5 & & \\
\hline Photodiode board (each) & 0.3 & $6 \times 10$ & 30 \\
\hline
\end{tabular}

Table 3. Estimates for mass, power dissipation and dimensions of the different PCBs (excluding margins and the associated mechanical support structure).

\section{FUTURE DIRECTIONS}

The design of the XIPE FW and calibration system is undergoing continuous development as it moves toward the end of the M4 assessment phase, and a number of different trade-offs are being studied and progressively frozen by the instrument team. Originally, a standard ratchet pinion gear, as that utilised for XMM-OM and Beagle-2 was assumed as a baseline. This design has evolved, due to the tight tolerances required in positioning the polarised Xray calibration source. Critically, the polarized source needs to be aligned within 3.5 arcmin with absolute repeatability, which moves the design into the direction of a worm wheel drive. Although this configuration has a lower TRL, these issues are being addressed early in the program and have been dealt with inside the team. The XIPE yellow book is currently required to be submitted to ESA by spring 2017, and the results of the first downselection of the M4 missions are expected by mid-2017. The down-selected candidate will then enter a definition phase, followed by a launch in 2026 .

MSSL-UCL is leading the FW design and provision. MSSL-UCL has a long standing experience in this, having produced filter wheels for several past missions: for instance, for the space instruments XMM/OM, Swift/UVOT, Beagle II/WAC, ExoMars/WAC ISO/LWS, and as well as for a ground based calibration system for JWST/NIRSPEC. Design and provision of Calibration sources are filters are led by IAPS and Tsinghua University, respectively.

The work of the MSSL is supported by the UK Space Agency. The Italian team is grateful for support by ASI, INFN and INAF. The XIPE team at the University of Geneva acknowledges the support of the Swiss State Secretariat for Education, Research and Innovation SERI, and ESA's PRODEX programme. The Polish Team acknowledges financial support from the Polish National Science Centre grant 2013/11/B/ST9/00234. We thank Thierry J-L. Courvoisier for support. 


\section{REFERENCES}

[1] Soffitta, P. et al., this volume (2016).

[2] Costa, E., Soffitta, P., Bellazzini, R., Brez, A., Lumb, N., and Spandre, G., "An efficient photoelectric Xray polarimeter for the study of black holes and neutron stars" Nature 411, 662 (2001).

[3] Bellazzini, R., Spandre, G., Minuti, M., Baldini, L., Brez, A., Cavalca, F., Latronico, L., Omodei, N., Massai, M. M., Sgro', C., Costa, E., Soffitta, P., Krummenacher, F., and de Oliveira, R., "Direct reading of charge multipliers with a self-triggering CMOS analog chip with $105 \mathrm{k}$ pixels at 50 um pitch" Nuclear Instruments and Methods in Physics Research A 566, 552 (2006).

[4] Bellazzini, R., Spandre, G., Minuti, M., Baldini, L., Brez, A., Latronico, L., Omodei, N., Razzano, M., Massai, M. M., Pesce-Rollins, M., Sgro, C., Costa, E., Soffitta, P., Sipila, H., and Lempinen, E., "A sealed Gas Pixel Detector for X-ray astronomy”, Nuclear Instruments and Methods in Physics Research A 579, 853 (2007). 